CROATICA CHEMICA ACTA CCACAA 53 (1) 51-68 (1980)

YU ISSN 0011-1643

CCA-1188

UDC 539.19

Author's Review

\title{
Potential Surface Studies of Open Shell Systems
}

\author{
P. Bischof \\ Institut für Organische Chemie der THD, Petersenstrasse 22, D 6100 Darmstadt
}

Received October 1, 1979

\begin{abstract}
The electronic structure of a large number of organic radicals was examined by using the developed unrestricted version of the semiempirical MINDO/3 method. A review of the obtained results is presented. The calculated heats of formation $\left(\Delta H_{\mathrm{f}}\right)$ are generally in good agreement with available experimental data. The estimated $\Delta H_{\mathrm{f}}$ values are usually too low $\sim 40 \mathrm{~kJ} / \mathrm{mol}$. This is not a serious drawback and had to be expected from the single determinant approach. However, it only shifts the origin of the energy scale. The relative stabilities of radical isomers are in most cases predicted quite correctly. Theoretical interatomic distances are in reasonably good accordance with experiment, Particular emphasis is placed on the discussion of the reactivity of radicals and reaction paths. Theoretical results are here of utmost importance because the elementary reactionsteps in radical rearrangements are extremely difficult to access by conventional experimental techniques. An original procedure for searching and verifying local minima and saddle points on the energy hypersurfaces is discussed. This approach is applied on the series of $\omega$-alkenyl radicals and their routes of cyclisations were throughly investigated. It was found that the experimental findings can be rationalised by a simple orbital overlap model which on the other hand leads to a natural link to the allowed/forbidden concept. These findings are corroborated by the available pieces of experimental evidence. An important outcome of our analyses is, that unlike for closed shell systems, the activated complexes of forbidden reactions for doublet states can be successfully described by single configurations and should therefore be accessible by appropriate semiempirical treatments.
\end{abstract}

The high reactivity of radicals makes it extremely difficult to study individual, elementary reaction steps by conventional experimental techniques. There is very often a number of competing reaction pathways which sometimes lead to ambiguous, in some cases even to contradictive interpretations of the obtained results.

Experimental computer chemistry ${ }^{1}$ provides an alternative course to study such processes, because each elementary reaction step can be simulated. Furthermore, such methods enable the chemist to investigate reactions which do not take place and the obtained results can teach him why they don't.

The aim of such calculations must be to provide a link between experiment and understanding which - if correct - is independent of the used model.

Note added in proof: While this work was in progress, the smallest system of $\omega$-alkenyl radicals $(n=2)$ has been published by $M$. J. S. D e w a $\mathrm{r}$ and $\mathrm{S}$. O 1 i v e 11 a $(J$. Amer. Chem. Soc. 101 (1979) 4958). It is very pleasing to note, that our calculations corroborate the predictions these authors reached from their results. 
The most commonly applied approach in this sense involves the study of the corresponding potential energy surfaces making use of Eyrings theory of the activated complex ${ }^{2}$.

In order to proceed this way, we need some means to meet the following two conditions:

1) A model which describes the potential surface as accurate and easily accessible as possible and

2) A mathematical procedure which allows to locate the essential structures with the least amount of numerical calculations.

Clearly, the first point calls for some reasonable compromise between reliability and computational effort. While ab initio methods beyond the Hartree Fock level have proven to be as accurate as experimental techniques to calculate intramolecular forces, these methods still are limited to rather small molecules. For systems of moderate or large size one is therefore forced to use some semiempirical approach to describe the potential energy surface.

Being aware of its limitations, MINDO $/ 3^{3}$ still seems to be the most reliable of these methods. Its main advantage as compared with other semiempirical models lies in the fact, that reasonable heats of formations as well as satisfactory geometries have been obtained for a large number of widely different molecules ${ }^{4}$.

Since the parameters used in this scheme hopefully balance the electronic correlation energy, its adequate application to open shell systems should be made using a restricted Hartree Fock version such as the half electron method". However, this method leads to serious problems in the gradient calculations which is a grave handicap in view of the second requirement mentioned above. We have therefore extended MINDO/3 to an unrestricted Hartree Fock version using the same set of parameters and formalism ${ }^{6}$.

A first important check for its suitability is then given by the comparison of the calculated properties of radicals in their ground state with the available experimental data or results of ab initio calculations. The latter are especially helpful in connection with equilibrium geometries, because experimental data are scarce.

Figure 1 shows the correlation between calculated and experimental heats of formation of a large series of radicals. It should be kept in mind, that the data represented in this plot have not been involved in the parametrisation procedure. Nevertheless, they are in reasonably good accordance with experiment $t^{6}$.

The largest deviations from the theoretical line with unit slope were found for radicals containing heteroatoms. Quite generally, the radicals are calculated to be too stable by an average of fourty $\mathrm{kJ} / \mathrm{mol}$. This tendency is not a serious shortcoming, because it only shifts the whole energy potential surface toward lower values. Our calculations showed on the other hand ${ }^{6}$, that the relative stabilities of isomer radicals are in most cases predicted quite correctly.

Survey of the ground state geometries shows, that the method provides reasonably good estimates of the structural parameters. 


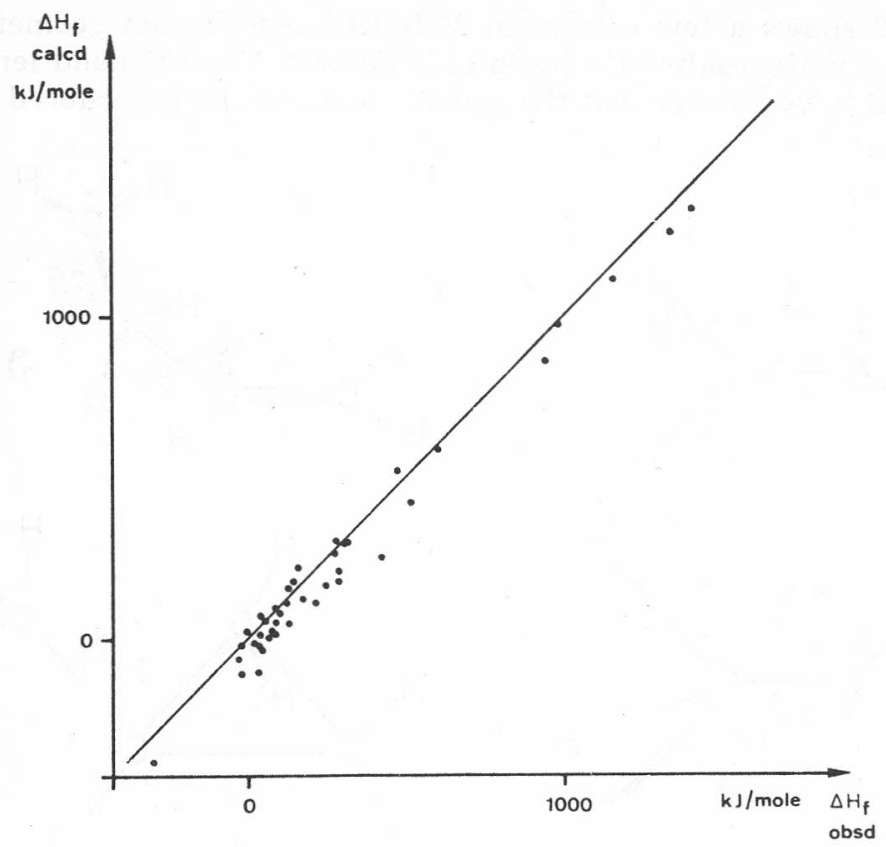

Figure 1. Correlation of calculated and experimental $\Delta H_{\rho}$ of radicals.

TABLE I

Calculated and Experimental Data of Diatomic Radicals (Bond Lengths in $A$, Wavenumbers in $\mathrm{cm}^{-1}$ )

\begin{tabular}{|c|c|c|c|c|c|}
\hline $\mathrm{R}^{\circ}$ & state & $r_{0}($ calc) & $r_{0}(\exp )$ & $v_{0}$ (calc) & $v_{0}(\exp )$ \\
\hline $\mathrm{CC}$ & ${ }^{3} \Pi_{1}$ & 1.261 & 1.312 & 1993 & 1641 \\
\hline $\mathrm{CCl}$ & ${ }^{2} \Pi$ & 1.641 & 1.642 & 903 & 846 \\
\hline $\mathrm{CH}$ & ${ }^{2} \Pi$ & 1.119 & 1.120 & 3347 & 2862 \\
\hline $\mathrm{CN}$ & $2 \Sigma^{+}$ & 1.132 & 1.172 & 2403 & 2069 \\
\hline $\mathrm{CO}^{+}$ & $2 \Sigma^{+}$ & 1.102 & 1.115 & 2396 & 2214 \\
\hline $\mathrm{CP}$ & ${ }^{2} \Sigma^{+}$ & 1.482 & 1.562 & 1590 & 1240 \\
\hline $\mathrm{HCl}^{+}$ & $\sqrt[2]{\Pi}$ & 1.275 & 1.315 & 2627 & 2675 \\
\hline $\mathrm{NN}^{+}$ & $2 \Sigma^{+} g$ & 1.050 & 1.116 & 2688 & 2207 \\
\hline NO & $2 \bar{\Pi}^{8}$ & 1.164 & 1.151 & 2117 & 1904 \\
\hline OO & $3 \bar{\Sigma}_{g}$ & 1.206 & 1.207 & 2107 & 1580 \\
\hline $\mathrm{OO}^{+}$ & ${ }^{2} \Pi_{g}$ & 1.137 & 1.123 & 2426 & 1876 \\
\hline $\mathrm{OH}$ & ${ }^{2} \Pi$ & 0.947 & 0.971 & 4027 & 3735 \\
\hline
\end{tabular}

Table I summarizes some calculated and spectroscopic data of diatomic radicals ${ }^{7}$. Although calculation and experiment compare reasonably well, the data disclose a general shortcoming of our method: All bond lengths are consistently too short, all stretching vibration wavenumbers too high ${ }^{8}$.

Diatomic radicals are definitely not the stronghold of any semiempirical treatment. However, the shown comparison proves, that the MINDO/3 results are at least realistic. 
Figure 2 shows a few calculated MINDO/3 equilibrium geometries which are compared with results of a initio calculations ${ }^{9}$. The $\mathrm{CC}$ bond lengths again are predicted to be shorter, but the general features are reproduced quite well.
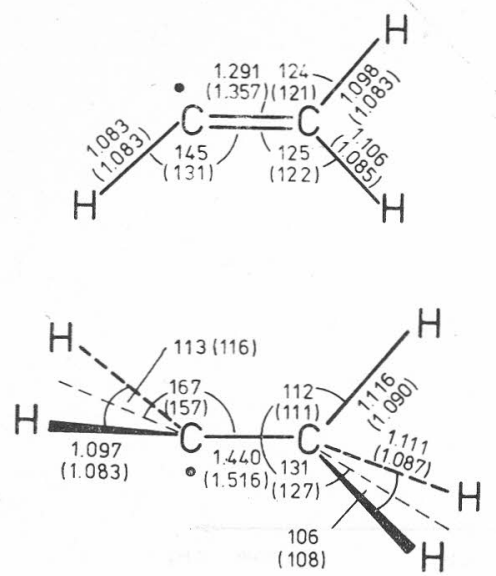
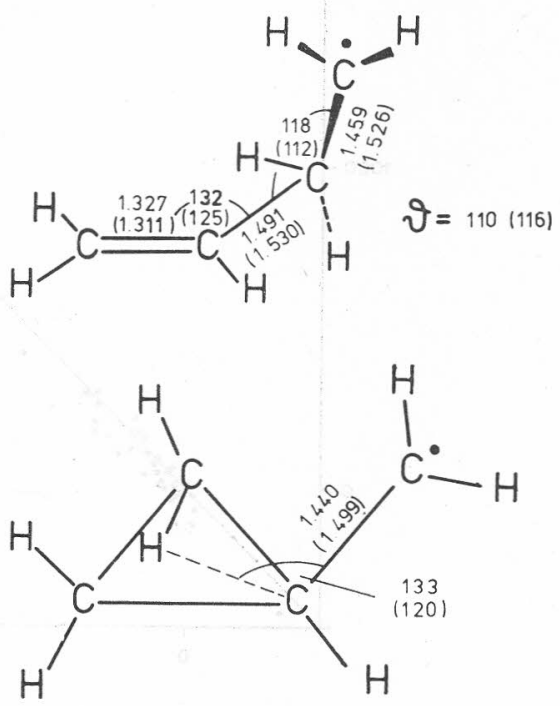

Figure 2. Comparison of predicted MINDO/3-UHF equilibrium geometries with results obtained by single configuration ab initio calculations. Only the optimised values of the latter are given in parentheses. (Ref. 32 )

Judging from the results discussed so far, we conclude, that the potential energy surface properties at least in the vicinity of predicted minimas are realistic and useful. As will be shown later, the energies of activated complexes are reasonable too.

We shall now turn to the second requirement mentioned above. As already anticipated, we wish to locate minimas and saddle points on the potential surface of interest.

Optimising a certain function or localising a minimum in a multidimensional space is a problem common to all fields of science. Accordingly, there is a large number of available procedures ${ }^{10}$. We have chosen the method developped by Fletcher and Powell ${ }^{11}$, because the calculation of the gradients in the MINDO/3-UHF scheme is trivial if the bond order matrix is kept constant.

This way, the equilibrium structures were located. They were verified to be true minimas by the standard procedure of calculating and diagonalising the force constant matrix $\mathbb{A}$. This was done in mass weighted cartesian coordinates which directly lead to normal vibrations or principal directions $q>$ in the way proposed by Wilson, Decius and Cross ${ }^{12}$.

Pulay has shown ${ }^{13}$, that in the calculation of second derivatives, the so called wave function forces cannot be neglected. This leads to the necessity of computing the matrix $\mathbf{A}$ by using a grid of points, each calculated by a full SCF calculation.

It turns out, that this verification needs about as much computer time as the search for the minima. However, the obtained data together with the 
located structures allows to estimate the standard entropies of the species by using statistical thermodynamics ${ }^{14}$.

Obviously, the contributions of internal rotors in this model are wrongly replaced by contributions of torsional vibrations with very low vibrational frequences. For radicals containing a large number of internal rotors, the method is therefore bound to fail.

Table II summarizes some calculated entropies "and compares them with "experimental " values. The term "experimental" is somewhat misleading, since the standard entropy of a radical cannot be measured. O'Neal and Benson have tabulated values obtained by the difference method (DM), based on the experimental values measured for the parent molecules and corrected for the missing hydrogen atom ${ }^{15}$.

TABLE II

Comparison of Calculated with "Experimental "Standard Entropies. Si is Calculated by Subtracting the Translational Part from $\mathrm{S}^{\circ}$. (All Values in $\mathrm{J} / \mathrm{kmol}$.)

\begin{tabular}{|c|c|c|c|c|c|}
\hline & \multicolumn{2}{|c|}{$S_{298}^{0}$} & \multicolumn{2}{|c|}{$S_{298}^{\mathrm{i}}$} & \multirow[b]{2}{*}{$\Delta$} \\
\hline & calc & $» \exp \ll$ & calc & $» \exp \ll$ & \\
\hline $\mathrm{CH}_{3}$ & 199 & 194 & 57 & 52 & 5 \\
\hline $\mathrm{C}_{2} \mathrm{H}_{5}$ & 253 & 248 & 103 & 98 & 5 \\
\hline $\mathrm{C}_{3} \mathrm{H}_{7}$ & 284 & 285 & 128 & 129 & -1 \\
\hline$i-\mathrm{C}_{3} \mathrm{H}_{7}$ & 294 & 283 & 138 & 127 & 11 \\
\hline$t-\mathrm{C}_{4} \mathrm{H}_{9}$ & 317 & 312 & 158 & 153 & 5 \\
\hline $\mathrm{CH}_{2} \mathrm{CHCH}_{2}$ & 262 & 260 & 105 & 103 & 2 \\
\hline $\mathrm{CH}_{2} \mathrm{CH}\left(\mathrm{CH}_{2}\right)_{2}$ & 323 & 312 & 165 & 154 & 11 \\
\hline
\end{tabular}

The tabulated standard entropies and their reduced internal contributions show, that the observed errors have about the magnitude of the calculated vibrational parts. Nevertheless, they might be used as a further corroboration, that the size of the radicals (and therefore their geometries) are quite accurate.

In addition, we used these standard entropies to roughly estimate the effects geometrical changes might have on the rate constants of radical rearrangement reaction $\mathbf{s}^{16}$.

The localisation of saddle points is a much more severe problem than localising minimas. A minimum on the potential energy surface $\mathrm{E}$ in most cases corresponds to a structure, which can be described by a classical Kekulé formula. This in turn is a useful guide to make a good starting point choice. On the other hand, such chemical intuition does not help much in estimating the structure of an activated complex.

McIver and Komornicky have developped a procedure which at first sight seems to be the method of choice ${ }^{17}$. Their theory is based on the fact, that minimas as well as saddle points are stationary points on the potential energy surface $E$. Hence, they are minimas on the gradient square surface $\sigma$. The value of $\sigma$ is given by:

$$
\sigma=\sum_{i=1}^{3 N-6}\left(\frac{\delta E}{\delta S_{i}}\right)^{2}=\langle g \mid g\rangle
$$


This way, the localisation of an activated complex is thus reduced to a simple" optimisation of the value of $\sigma$. This can in principle again be performed using the Fletcher-Powell procedure. However, the calculation of the derivatives of $\sigma$ involves the determination of the force constant matrix $\mathbf{A}$, because

or in vector notation

$$
\frac{\delta \sigma}{\delta S_{\mathrm{i}}}=\underset{\mathrm{j}=1}{2 \mathrm{~N}-6} A_{\mathrm{ij}} \frac{\delta E}{\delta S_{\mathrm{j}}}
$$

$$
\left|\sigma^{\prime}\right\rangle=2 \mathbf{A}|g\rangle
$$

As already indicated, the calculation of $\mathbf{A}$ is a tedious piece of work. Since this has to be done at each point reached in the optimisation procedure, the localisation of the desired saddle points became hopelessly slow.

We therefore turned to an alternative procedure which proved to be superior as far as the computer time consumption is concerned. The method can be described as follows: Find the saddle point as naive and quick as possible and spend more time in verifying its nature to be the true saddle point of interest.

This is performed in five steps:

1) The reactant structure is optimised. From the geometry defining variables $a_{i}$ (bondlengths, bondangles and dihedral angles) the one is chosen as a trial reaction coordinate $\alpha$, which apparently changes its value the most as the reactant continuously turns into the product. In most cases, $\alpha$ is the length of the bond being broken or formed in the process.

2) This variable is then varied in small increments at which points the energy is optimised with respect to all other variables $\alpha_{\mathrm{i}}$ thus making all first derivatives zero,

$$
\left(\frac{\delta E}{\delta a_{\mathrm{i}}}\right)_{\alpha}=0
$$

within a specified accuracy. At this geometry $|a\rangle$, the derivative of $E$ with respect to $\alpha$ is calculated.

This "step search"« is repeated, until the geometry is located, at which

$$
\left(\frac{\delta E}{\delta \alpha}\right) \underset{a_{\mathrm{i}} \neq \alpha}{=0}
$$

The geometry obtained this way shall be denoted $|t\rangle$. McIver has pointed out $^{17}$, that this structure does not necessarily have to be a saddle point. We therefore applied the following tests:

3) The force constant matrix $\mathbf{A}$ was calculated and the $3 \mathrm{~N}-6$ principal directions $\mid q>$ obtained as its eigenvectors. It was checked that one and oniy one calculated eigenvalue was negative. The principal direction $\mid q^{-}>$belonging to this negative eigenvalue is in fact »the way over the hill « and has been called the transition vector ${ }^{17}$ or the intrinsic reaction coordinate (IRC) ${ }^{18}$.

4) The first derivatives of $E$ with respect to the principle directions $|q\rangle$ were calculated by finite differences insuring that they all were below the convergence criteria.

Taking for granted, that these checks are sufficient to prove, that $|t\rangle$ really is a saddle point, there still remains the problem to find, whether or 
not it is the only activated complex along the reaction path. This was done in the final step:

5) The structure $\mid t>$ was modified by the transition vector $\mid q^{-}>$which led to two nearly identical structures $\mid t>^{+}$and $\mid t>^{-}$as

and

$$
|t\rangle^{+}=|t>+\lambda| q^{-}>
$$

$$
|t\rangle^{-}=\left|t>-\lambda ; q^{-}\right\rangle \text {. }
$$

The factor $\lambda$ was chosen as 0.01 . The obtained geometries were then transformed back to the internal coordinate system $|a\rangle$ and the structures were optimised. $|t\rangle^{+}$converged to the structure of the product (if the reaction was a one step reaction), $\mid t>^{-}$to the starting point reactant.

Sometimes, however, the relaxed structure of $|t\rangle^{-}$was quite different from that of the starting point. In these cases, the starting structure and the relocated reactant structure were always found to be local minimas separated by small activation energies such as rotational barriers.

The program system which we just described is - apart from the spin unrestricted modification of $\mathrm{MINDO} / 3$ - identical to the one used to study reactions on a singlet surface.

Its application to a reaction of interest leads to a scheme as the one shown in Figure 3. This picture shows as an example the rearrangement norbornenyl-nortricyclylradical, which has been studied experimentally by Giese $^{19}$ The arrows shown in the plot of the activated complex correspond to the nuclear displacements along the intrinsic reaction coordinate.
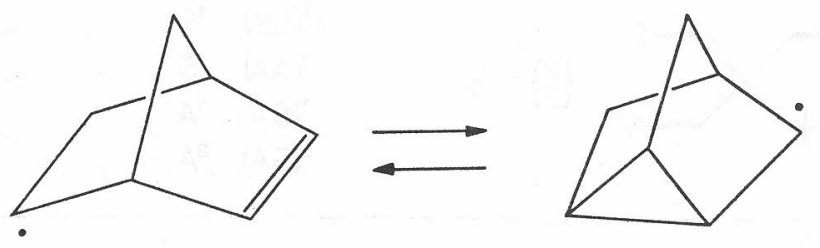

Giese has concluded from his measured kinetic data that

1) both reactants have about the same energy, the norbornenylradical being at most ${ }^{20} 8 \mathrm{~kJ} / \mathrm{mol}$ more stable than the nortricyclylradical and

2) the activation energy amounts to $48 \mathrm{~kJ} / \mathrm{mol}$ for the forward, to 40 $\mathrm{kJ} / \mathrm{mol}$ for the reverse reaction.
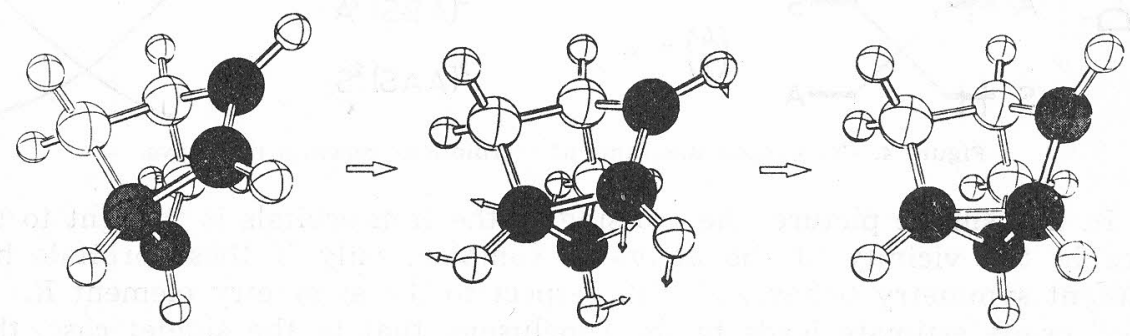

Figure 3. Calculated structures of the norbornenyl/nortricyclylradical rearrangement. 
MINDO/3-UHF predicts both radicals to have the same heat of formation, the activation energy is calculated to be $48 \mathrm{~kJ} / \mathrm{mol}$.

This reaction is an example of a widely observed type of radical rearrangement: It is a homoallyl/cyclopropyl carbinyl rearrangement. The shaded atoms in Figure 3 indicate the carbon atoms which form the parent system. We shall return to a detailed study of the parent reaction later on.

An important aspect we have not mentioned so far might serve as a further justification for the application of »experimental computer chemistry « to radical rearrangements.

For singlet molecules, the Woodward Hoffmann rules ${ }^{21}$ provide a very useful way to predict the ease with which a reaction will proceed and what stereochemical consequences are to be expected. Unfortunately, these rules are not conclusive for doublet systems ${ }^{22}$.

However, the analogue way to investigate reactions could explain expected differences in the potential surface behaviour of open shell as compared with singlet systems.

Such differences are thought to be especially pronounced for so called »forbidden « reactions, while for »allowed « reaction paths, smooth, well behaved potential surfaces are expected for either type of reaction.

It is well understood, that the activated complex of a forbidden reaction path for a system with an even number of electrons cannot be described by a single configuration. This seems to be different for radical systems. The correlation diagrams for both are compared in Figure 4.

Orbitals Components Configurations States
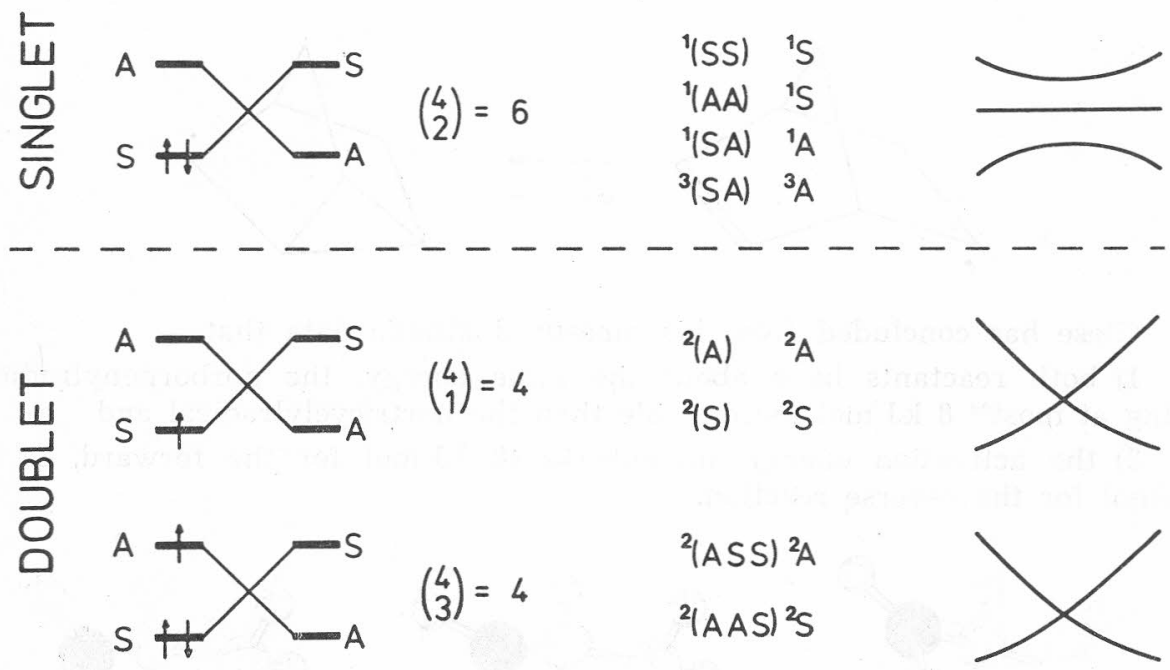

Figure 4. Correlation diagrams of "forbidden " reaction pathways.

In this simple picture, the crossing of the frontorbitals is thought to take place in the vicinity of the activated complex, only if these orbitals have different symmetry behaviour with respect to the symmetry element $R$.

A crude estimate leads to the conclusion, that in the singlet case, three singlet (and one triplet) configurations are of comparable energy, while in 
both doublet cases, only two configurations of different symmetries have to be considered. Hence, in the former, configuration interaction might lead to an avoided crossing while in the latter, the surfaces should cross indeed ${ }^{23}$.

Evidently, the expected electronic structure of the activated complex in 'the doublet case resembles that of a Jahn Teller state radical and detailed calculations of such systems have led us to expect a surface behaviour as the one shown in Figure 5.23. In this plot, $Q_{1}$ corresponds to a symmetrical reaction coordinate, $Q_{2}$ to the Jahn Teller distortion which is necessarily antisymmetric with respect to the symmetry element $R$.

This qualitative scheme therefore predicts, that a »forbidden « disrotatory radical rearrangement proceeds via two enantiomer reaction paths.

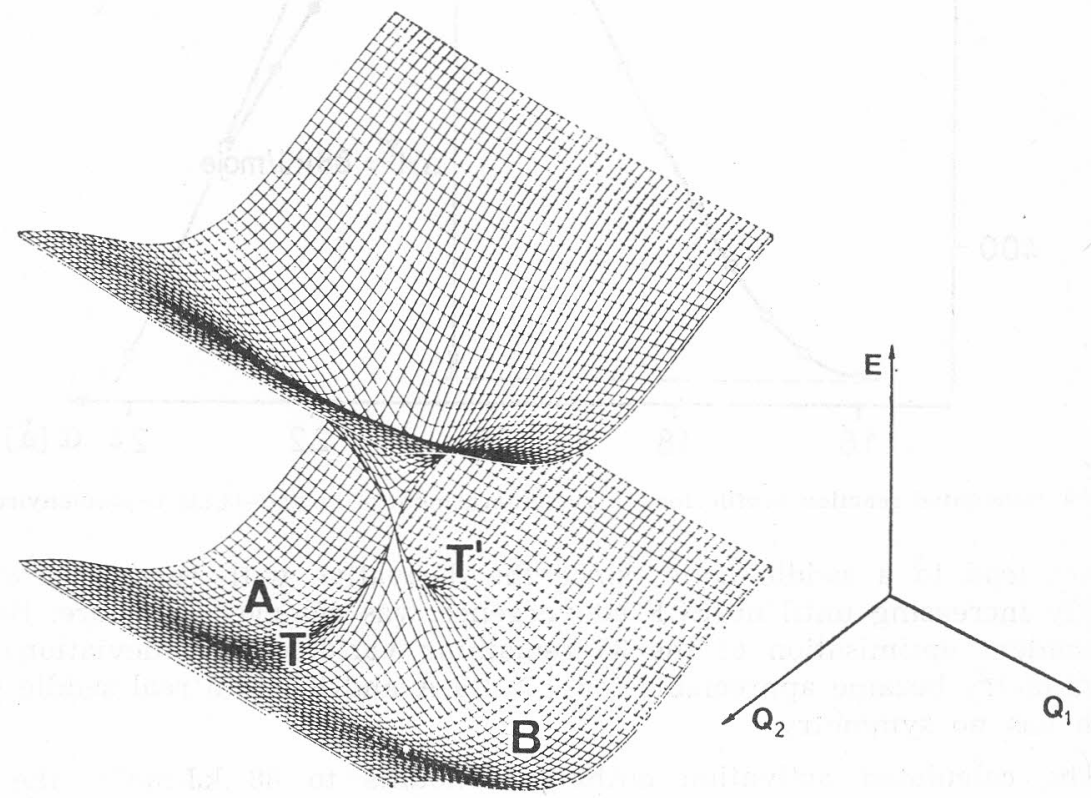

Figure 5. Model potential surface of a sforbidden« doublet reaction.

We wish to illustrate this point by a specific example, for which experimental data are available.

Sustmann ${ }^{24}$ has investigated the rearrangement of the bicyclo-[3,2,0]-heptadienylradical which leads to the tropylradical in an adamantane matrix by ESR and found a free activation energy of $90 \mathrm{~kJ} / \mathrm{mol}$ at $323 \mathrm{~K}$.
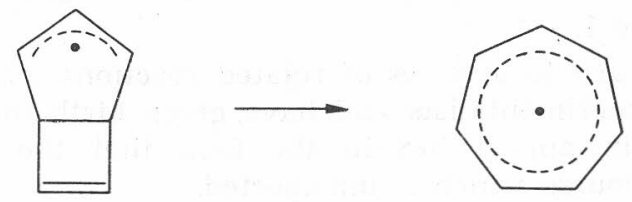

Since the search for the activated complex led to some problem, the calculated reaction profile is shown in Figure 6. As the trial reaction coordinate, we have chosen the bondlength $\mathrm{C}^{1} \mathrm{C}^{5}$. Enforcing $\mathrm{C}_{\mathrm{S}}$ symmetry, the search 


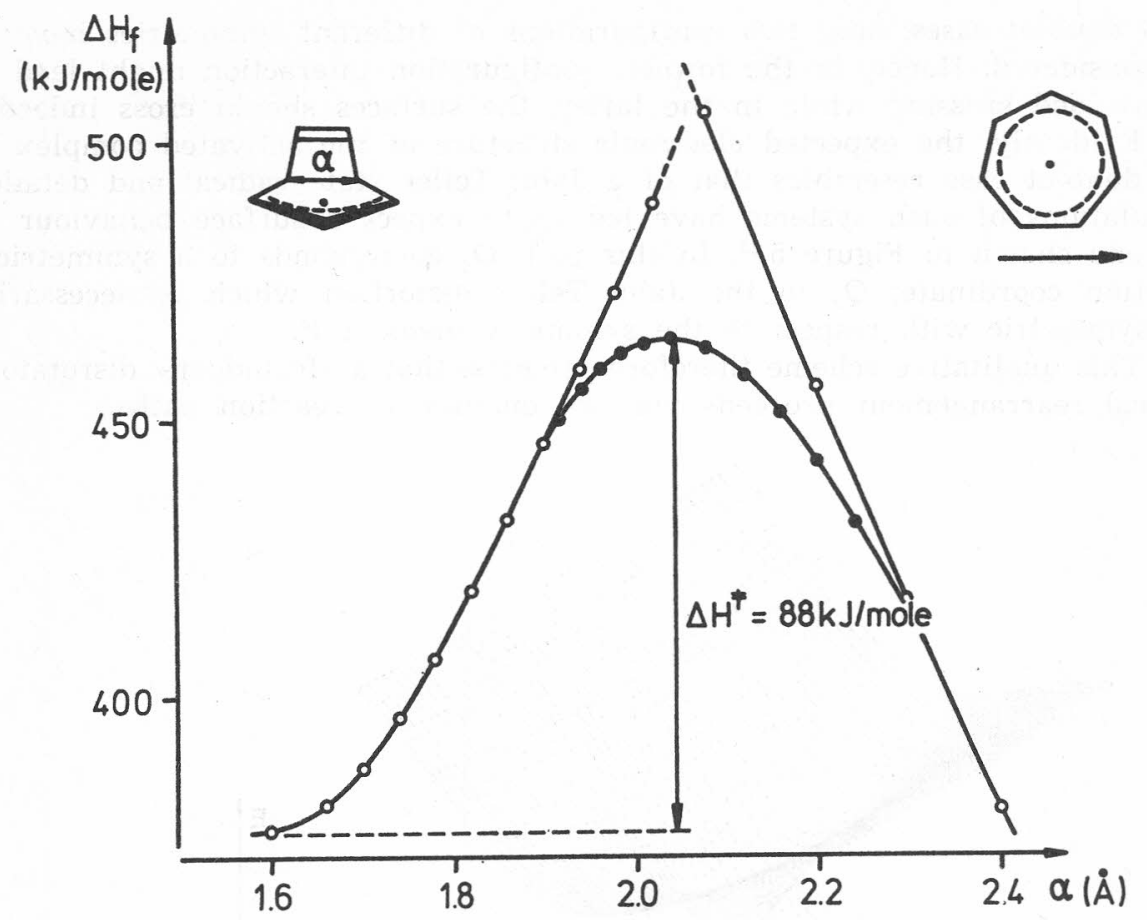

Figure 6. Calculated reaction profile for the ringenlargement of bicyclo-[3,2,0]-heptadienylradical.

did not lead to a saddle point, since the derivative with respect to $\alpha$ was steadily increasing until no SCF convergence was achieved anymore. By the independent optimisation of the 35 remaining variables, the deviation from $\mathrm{C}_{\mathrm{S}}$ symmetry became appreciable for $\alpha>1.9 \AA$ and led to a real saddle point which has no symmetry.

The calculated activation enthalpy amounts to $88 \mathrm{~kJ} / \mathrm{mol}^{23}$, the free activation enthalpy $\Delta G_{323} \neq$ (which includes the estimated small change in entropy) to $86 \mathrm{~kJ} / \mathrm{mol}^{25}$. The calculated structures are shown in the usual way in Figure 7.

The given examples show, that »allowed « as well as »forbidden « reaction pathways can be treated reasonably well with MINDO/3-UHF. We are aware, that the tremendous numerical accuracy encountered above is probably due to a fortuitous cancellation of errors, because the standard deviation of radicals' heats of formation in their equilibrium structure is much larger as already anticipated in Figure 1.

We shall now turn to a series of related reactions which have attracted interest of many experimentalists and have given birth to a vast number of papers ${ }^{26}$. Their main appeal lies in the fact, that the observed reaction pathways follow a course which is unexpected.

Radicals add very rapidly to molecules containing a $\mathrm{CC}$ double bond. This intermolecular reaction is known to involve a small activation barrier in the order of twenty $\mathrm{kJ} / \mathrm{mol}$. Theory ${ }^{27}$ is on line with experiment ${ }^{28}$, that in case 


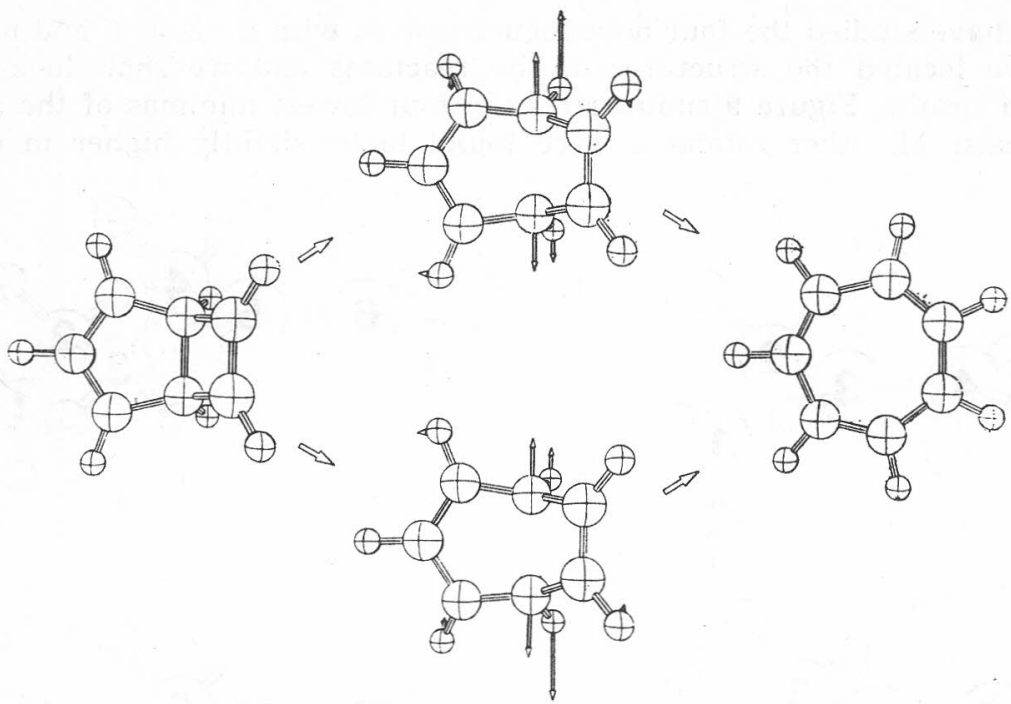

Figure 7. Calculated structures of the ringenlargement of bicyclo-[3,2,0]-heptadienylradical.

of relatively nonpolar additions, this reaction takes the Markownikow route which leads to the thermodynamically more stable products.

If the double bond and the radical center are part of the same molecule, the addition (which in this case is a cyclisation) proceeds exclusively along the alternative anti-Markownikow course if the molecule is sufficiently small ${ }^{29}$, while for larger systems, the "normal « Markownikow cyclisation is observed as well ${ }^{30}$. The parent system is illustrated in Figure 8. For each number of methylene groups $\mathrm{n}$, there are three types of reactants and likewise three possible reaction pathways $A, M$ and $S$ which have to be considered in the study of the corresponding potential energy surfaces.<smiles>C=CCCCC</smiles>

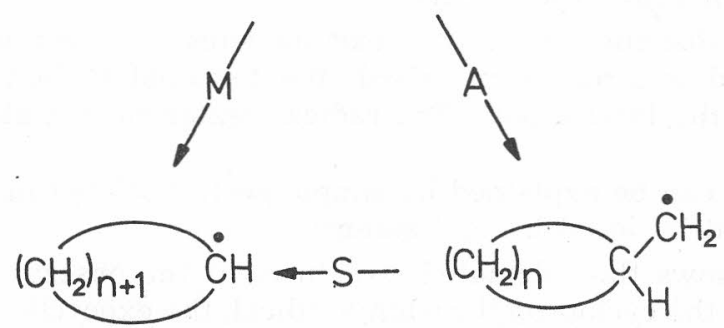

Figure 8. Parent system of w-alkenylradical cyclisations.

Since in this notation, the number $\mathrm{n}$ includes the radical center, the smallest member of the series is the homoallylradical $(n=2)$, where the two functional groups of the open chain $\omega$-alkenylradical are still separated by one linking methylene group. 
We have studied the four homologue systems with $n=2,3,4$, and $n=5^{16,31}$. First, we located the structures of the reactants and we shall look at the obtained results. Figure 9 summarizes the four lowest minimas of the $\omega$-alkenylradicals. All other rotamers were found to be slightly higher in energy.
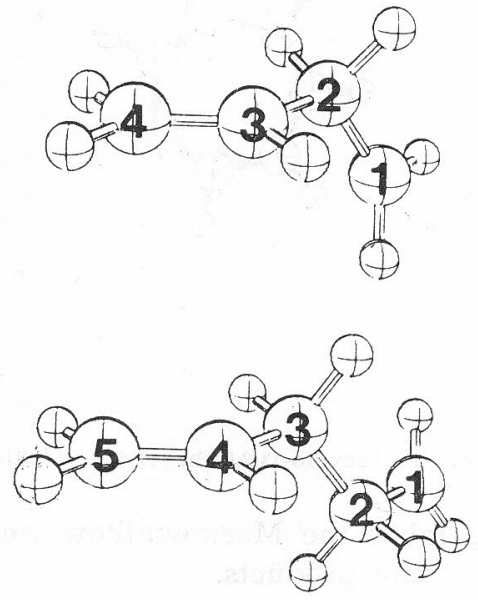
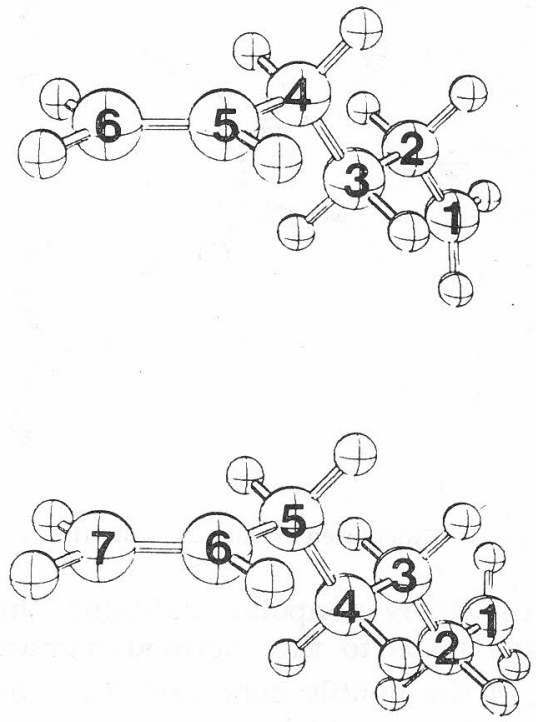

Figure 9. Equilibrium structures of w-alkenylradicals.

Apparently, the structures have very much in common: They consist of an all trans-methylene-chain substituting the terminal double bond in gauche conformation, while the radical center plane at the other end in all radicals intersect the adjacent methylene group.

As already shown in Figure 2, the structure of the smallest member of the series has been calculated by ab initio ${ }^{32}$, Hehre's result being essentially identical with ours except for the conformation of the radical site, where the two results contradict. Our prediction is strongly supported by experimental findings based on ESR experiments ${ }^{29}$.

The charge distribution at the reaction sites is worth to be mentioned: The double bond is strongly polarised, the terminal carbon atom being the negative end of the local dipole. The radical center carbon atom is negatively charged too.

Both effects can be explained by simple perturbational models, the former has been analised in detail by Hoffmann ${ }^{33}$.

Figure 10 shows the calculated structures of the observed cycloalkyl carbinylradicals. In the cyclopropyl carbinylradical, the exocyclic methylene group assumes the perpendicular conformation, while in all other members of the series, the equilibrium conformation is parallel with the ring of the cycloalkyl substituent.

In the cyclopropyl carbinylradical, the cyclopropyl substituent provides a very effective orbital to conjugate with the localised $\varrho$ orbital at the radical center: The antisymmetric Walsh orbital ${ }^{34}$. 

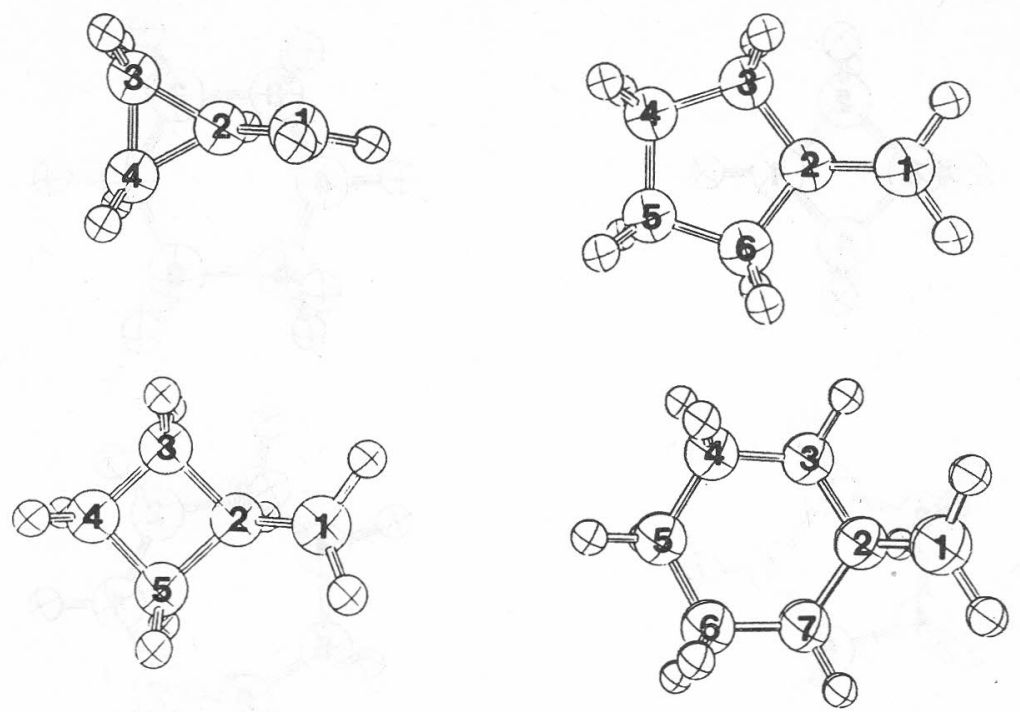

Figure 10. Equilibrium structures of cycloalkyl carbinylradicals.

The resulting conjugation leads to a marked stabilisation of the radical which more than outbalances the repulsion between the two eclipsed hydrogenatoms. For any higher homologue, such an effect is much smaller because the corresponding Walsh orbitals are too low in energy.

This leads to a relatively high rotational barrier around the exocyclic $\mathrm{CC}$ bond of $7 \mathrm{~kJ} / \mathrm{mol}$ for the cyclopropyl carbinylradical, while in all other cases, the corresponding values were less than $2 \mathrm{~kJ} / \mathrm{mol}$.

To complete the summary of reactant structures, the calculated geometries of the cycloalkyl radicals are shown in Figure 11. They show no peculiar aspects wortwhile to be discussed in this context.

The calculated heats of formation of the reactants predict, that in all cases, the cycloalkylradical is the most stable. The $\omega$-alkenylradical is the least stable with the exception of the smallest system, where the ringstrain of the threemembered ring reverses the ordering of the $\omega$-butenyl and the cyclopropyl carbinyl radical.

This is on line with experiment ${ }^{15}$, although the absolute heats of formation are all too low as anticipated earlier.

The outcome of the cyclisation reaction is not governed by the stability of the products. This is a fact which follows from the experimental findings and which is reproduced by our calculations.

The calculated activation parameters are summarized in Table III. For the activation entropies of route $A$ and route $M$ cyclisations, we needed to estimate the standard entropies of the open chain $\omega$-alkenylradicals, which for the reasons given above should not be obtained by the direct calculations.

Looking at the standard entropies of linear alkanes ${ }^{35}$, it can be found, that the lengthening of the chain by one methylene group raises the standard entropy consistently by $40 \mathrm{~J} / \mathrm{kmol}$. Therefore, we calculated the standard entropy of the smallest member of the series, adding $40 \mathrm{~J} / \mathrm{kmol}$ to each next higher homologue. 

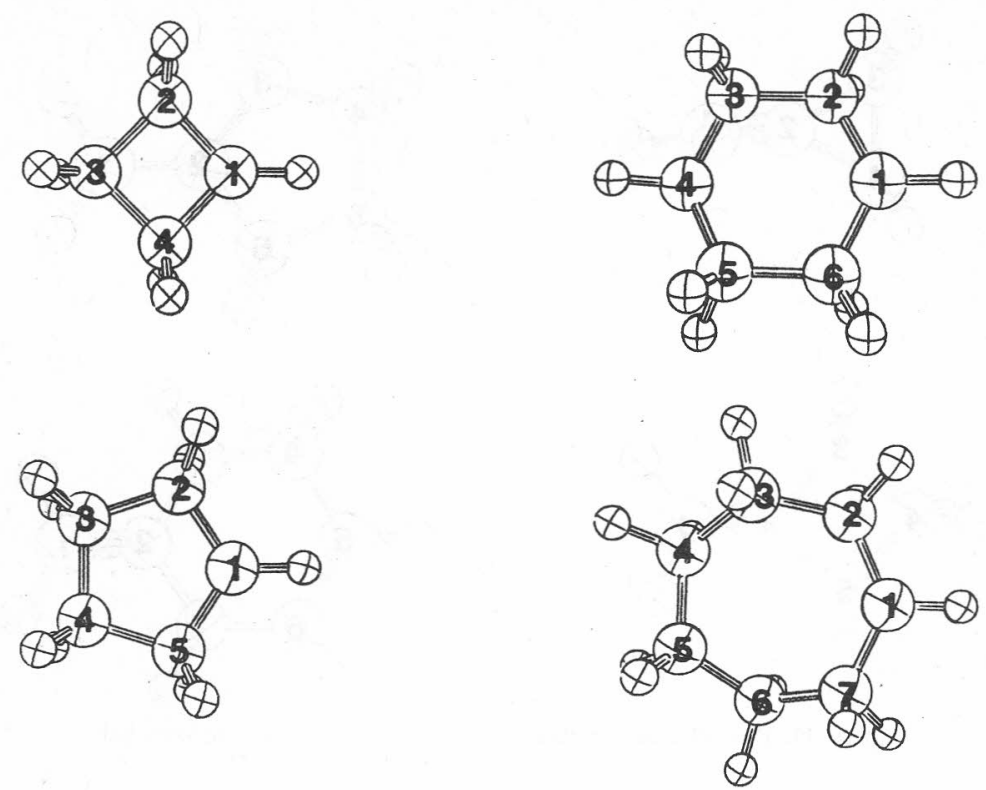

Figure 11. Equilibrium structures of cycloalkylradicals.

TABLE III

Comparison of Energies, Entropies and Free Energies of Activation for the Three Investigated Types of Rearrangements. $\left(\triangle \mathrm{H} \neq\right.$ and $\triangle \mathrm{G}_{298} \neq$ in $\mathrm{kJ} / \mathrm{mol}$, $\Delta \mathrm{S}_{298} \neq$ in $\mathrm{J} / \mathrm{kmol}$.)

\begin{tabular}{cccc|rcc|ccc}
\hline & \multicolumn{3}{c|}{ Route $M$} & \multicolumn{3}{c|}{ Route $A$} & \multicolumn{3}{c}{ Route $S$} \\
\hline $\mathrm{n}$ & $\Delta H \neq:$ & $\Delta S_{298} \neq$ & $\Delta G_{298} \neq$ & $\Delta H \neq$ & $\Delta S_{298} \neq$ & $\Delta G_{298} \neq$ & $\Delta H \neq$ & $\Delta S_{298} \neq$ & $\Delta G_{298} \neq$ \\
\hline 2 & 124 & -39 & 135 & 52 & -34 & 62 & 136 & -15 & 140 \\
3 & 91 & -46 & 105 & 76 & -43 & 88 & 139 & -22 & 146 \\
4 & 67 & -47 & 81 & 67 & -34 & 77 & 136 & -22 & 142 \\
5 & 65 & -60 & 82 & 77 & -60 & 95 & 136 & -19 & 142 \\
\hline
\end{tabular}

From the calculated activation enthalpies the following trends are apparent:

1) The route $A$ activation enthalpy slowly increases,

2) The route $M$ activation enthalpy at first strongly decreases while

3) The activation enthalpies for route $S$ are all very high and do not change significantly with increasing size of the system.

From these values, we conclude, that for $n<4$ the anti-Markownikow pathway strongly dominates the outcome of the cyclisation, while for $n>4$ both cyclisation mechanisms are competitive, the Markownikow route becoming more and more dominant, as the system becomes large.

Furthermore, a direct rearrangement via route $S$ is highly unlikely. This pathway corresponds to a 1,2 alkyl shift which indeed has never been observed in saturated radical systems ${ }^{30}$. 
All these results have been fully corroborated by experiment ${ }^{26}$. The reasons for these trends can be found in the structures of the corresponding saddle points.

We shall begin the discussion with the route $A$ activated complexes. The obtained structures are shown in Figure 12, where the arrows display the intrinsic reaction coordinates.
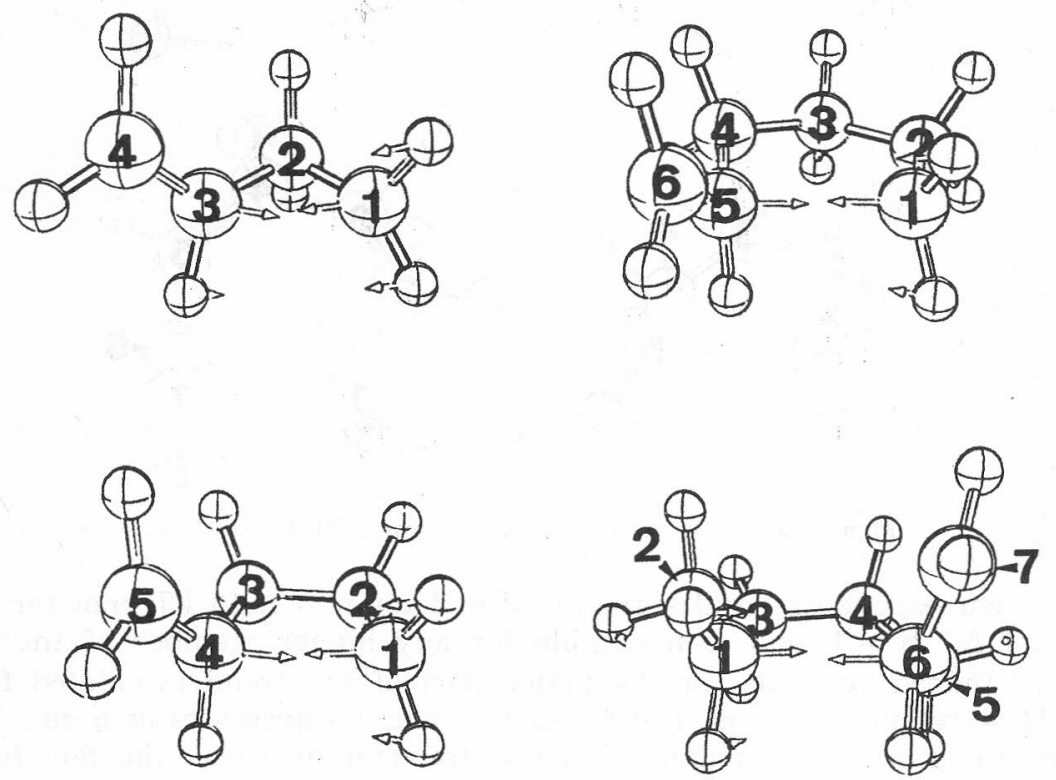

Figure 12. Activated complexes for route $A$ cyclisations.

Note, that in all cases, the terminal double bonds are essentially intact and that the transition vectors resemble a simple stretch vibration.

This is quite different in the saddle points associated with route $M$ shown in Figure 13.

The formerly simple intrinsic reaction coordinates in these cases are strongly coupled with a rotation of the methylene group which in the $\omega$-alkenylradical was part of the terminal double bond. The rotation diminishes, as the size of molecule increases.

This rotation indicates a dominant decoupling effect of the double bond in the activated complex of small systems. Such a torsional twist is associated with a large increase in energy.

The amount of the double bond decoupling is given by the corresponding dihedral angle between the $\mathrm{p}$ orbitals which participate in the $\pi$ bond.

These angles $\alpha$ were calculated to be $28^{0}$ for $\mathrm{n}=2,18^{0}$ for $\mathrm{n}=3,9^{0}$ for $\mathrm{n}=4$, and $5^{0}$ for $\mathrm{n}=5$.

In a simple perturbational picture, the associated raise in energy $\Delta E$ can be estimated, if we assume that the $\pi / \pi^{*}$-split is proportional to the $\mathrm{p} / \mathrm{p}$ overlap and that the complete decoupling of ethylene amounts to $260 \mathrm{~kJ} / \mathrm{mol}^{36}$. 

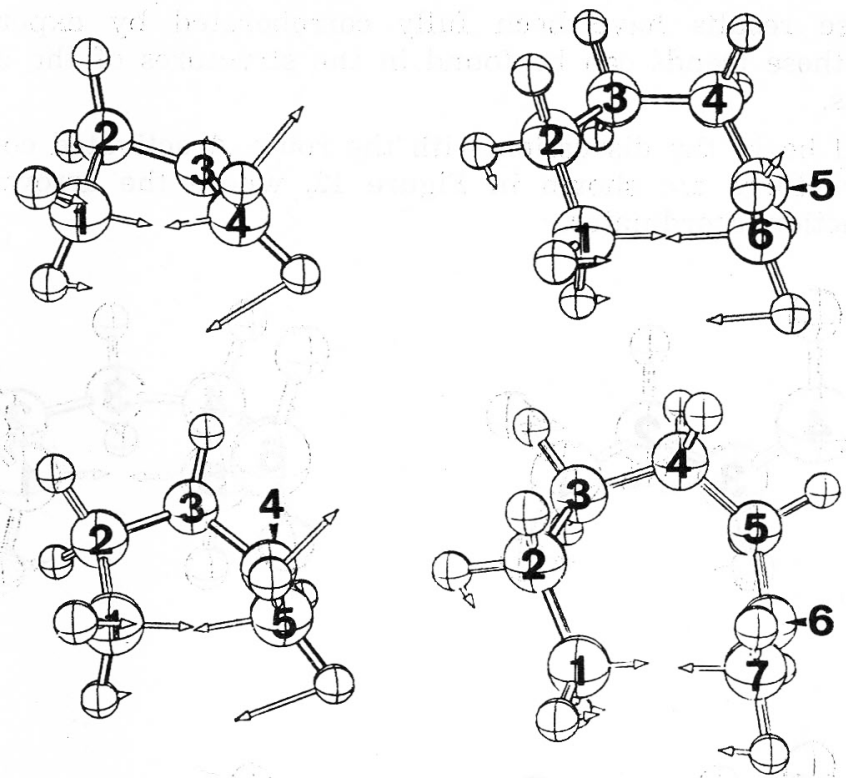

Figure 13. Activated complexes for route $M$ cyclisations.

The corresponding results are $31 \mathrm{~kJ} / \mathrm{mol}$ for $\mathrm{n}=2,13 \mathrm{~kJ} / \mathrm{mol}$ for $\mathrm{n}=3$, $3 \mathrm{~kJ} / \mathrm{mol}$ for $\mathrm{n}=4$, and is negligible for any higher member of the series.

This indeed accounts for the major part of the trend calculated for the route $M$ activation energies. The decoupling angle $\alpha$ decreases as $\mathrm{n}$ gets larger, because the radical gains more dynamic freedom to attack the double bond in a better fashion.

An entirely different point of view is possible, if the series is extended to the smallest system, where $\mathrm{n}=1$.

This extension was initiated by the appearance of the activated complex. of the Markownikow cyclisation of the $(1)$-butenylradical, which reminded us very much of the activated complex we calculated for the allyl/cyclopropyl rearrangement ${ }^{37}$. The two calculated structures are compared in Figure 14.
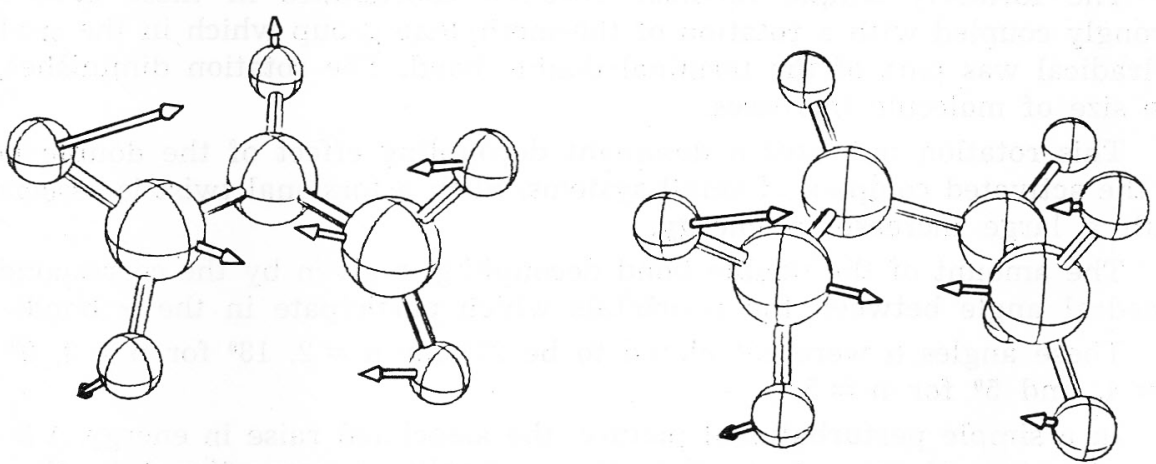

Figure 14. Comparison of activated complexes of route $M$ cyclisation of $\omega$-butenviradical with the allyl/cyclopropyl rearrangement. 
If the route $\mathrm{A}$ and route $M$ cyclisations are formulated accordingly, it turns out, that the former is simply a rotation of the terminal methylene group, thus leading to the "two-membered " ring, while the latter corresponds to the classical case of a forbidden radical rearrangement: The allyl/cyclopropyl rearrangement. Updating the calculated activation energies finally leads to Figure 15, which turns the formerly unexpected outcome of $\omega$-alkenyl radical cyclisations into the most natural, well understood experimental fact.

This extension, which in the backward glance is trivial, has to our knowledge never been made so far. We definitely believe, that such insights are the real destinations which should be reached by »experimental computer chemistry«.

Numerical accuracy of the method in this view is not a crucial point, but helps to trace the reasons for the observed trends to the correct origins.

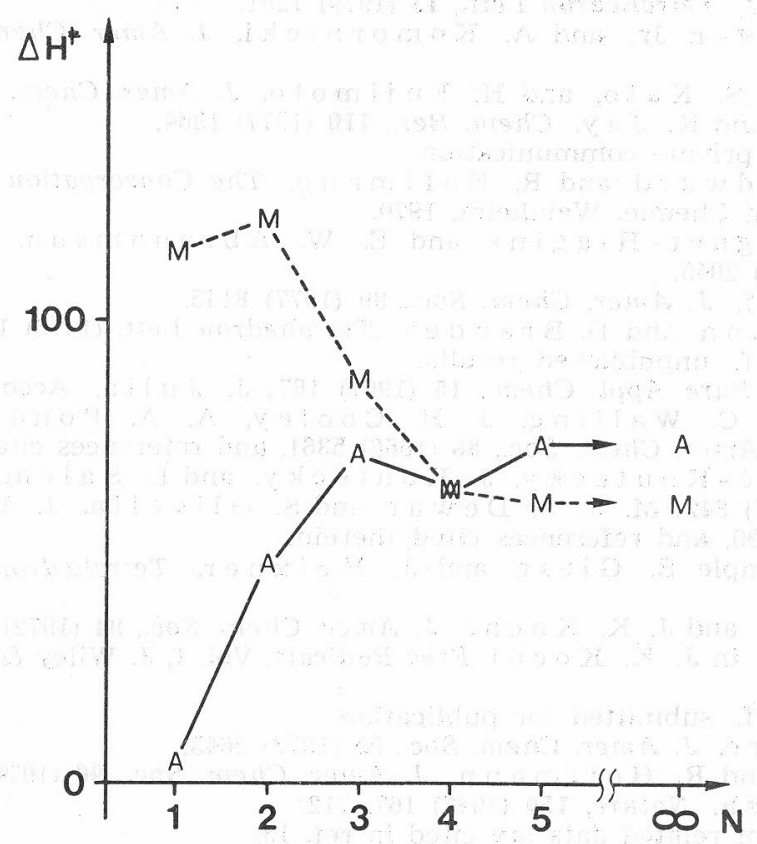

Figure 15. Correlation of activation enthalpies of route $A$ and route $M$ cyclisations with increasing number of methylene groups $n . n=\infty$ corresponds to the intermolecular radical addition.

Acknowledgment. - The author is grateful to Professor C. Rüchardt, who initiated. the study of $\omega$-alkenylradical cyclisation mechanisms by a motivating discussion, Mrs. P. Hofmann for patiently typing the manuscript.

\section{REFERENCES}

1. M. J. S. D ew a r, Chem. Brit. 11 (1975) 97.

2. H. Eyring, J. Chem. Phys. 3 (1935) 107.

3. R. C. B ing h a m, M. J. S. D e w a r, and D. H. Lo, J. Amer. Chem. Soc. 97 (1975) 1285.

4. R. C. Bing h a m, M. J. S. Dew a r, and D. H. Lo, J. Amer. Chem. Soc., 97 (1975) 1294, 1302, 1307; M. J. S. D e w a r, D. H. L o, and C. A. R a m s d e n. ibid, 97 (1975) 1311. 
5. M. J. S. Dewar, J. A. Hashmall, and C. G. Venier, J. Amer. Chem. Soc., 90 (1968) 1953.

6. P. B is c h of, J. Amer. Chem. Soc. 98 (1976) 6844.

7. Experimental results taken from G. He r z b e r g, Spectra of Diatomic Molecules, Van Nostrand, Prince: on, N. J., 1950.

8. M. J. S. D e war and G. P. Ford, J. Amer. Chem. Soc. 99 (1977) 1685.

9. W. A. Lathan, W. J. Hehre, and J. A. Pople, J. Amer. Chem. Soc., 93 (1971) 808.

10. See for example: R. Fletcher, Optimization, Acad. Press, London (1969).

11. R. Fletcher and M. J. D. Powell, Comput. J., 6 (1963) 163.

12. E. B. Wilson, J. C. Decius, and P. C. Cross, Molecular Vibrations, McGraw Hill, N. Y. 1955.

13. P. Pulay, Molec. Phys., 17 (1969) 197.

14. F. N. Godnew, Berechnungen thermodynamischer Funktionen aus Moleküldaten, Deutscher Verlag der Wissenschaften, Berlin (1963).

15. H. E. O' Neal and S. W. Benson in J. K. Kochi, Free Radicals, Vol. II, (1973) 275, J. Wiley \& Sons, N. Y. 1973.

16. P. B is ch of, Tetrahedron Lett., 15 (1979) 1291.

17. J. W. Mc I ver Jr. and A. Komornicki, J. Amer. Chem. Soc., 94 (1972) 2625.

18. K. Fukui, S. Kato, and H. Fujimoto, J. Amer. Chem. Soc., 97 (1975) 1.

19. B. Gie se and K. J a y, Chem. Ber., 110 (1977) 1364.

20. B. Gi e s e, private communication.

21. R. B. Wood ward and R. Hof $\mathrm{fmann}$, The Conservation of Orbital Symmetry, Verlag Chemie, Weinheim, 1970.

22. H. C. Longuet-Higgins and E. W. A.brahamson, J. Amer. Chem. Soc., 87 (1965) 2045.

23. P. B is ch of, J. Amer. Chem. Soc., 99 (1977) 8145.

24. R. Sustmann and D. B randes, Tetrahedron Lett. (1976) 1791.

25. P. Bis ch of, unpublished results.

26. M. Julia, Pure Appl. Chem., 15 (1967) 167; J. Julia, Accounts Chem. Res., 4 (1971) 386; C. Walling, J. H. Cooley, A. A. Ponaras, and E. J. $\mathrm{R}$ a c a h, J. Amer. Chem. Soc., 88 (1966) 5361, and references cited therein.

27. V. B on a čić-Koutecky, J. Kout e cky, and L. Sale m, J. Amer. Chem. Soc., 99 (1977) 842; M. J. S. D e w a r and S. Olivella, J. Amer. Chem. Soc., 100 (1978) 5290, and references cited therein.

28. See for example B. Gies e and J. M e ixner, Tetrahedron Lett., 32 (1977) 2779.

29. D. J. Edge and J. K. Koch i, J. Amer. Chem. Soc., 94 (1972) 7695.

30. J. W. Wilt in J. K. Koch i Free Radicals, Vol. I, J. Wiley \& Sons, New York (1973).

31. P. B is ch of, submitted for publication.

32. W. J. H e h r e, J. Amer. Chem. Soc., 95 (1973) 2643.

33. L. Libit and R. Hof fma n n, J. Amer. Chem. Soc., 96 (1974) 1370.

34. A. D. W a ls h, Nature, 159 (1947) 167, 712.

35. A summary of related data are cited in ref. 15 .

36. M. H. W o o d, Chem. Phys. Lett., 24 (1974) 239.

37. G. Friedrich and P. Bischof, to be published.

\section{SAŽETAK}

\section{Istraživanja potencijalnih ploha sustava $\mathrm{s}$ otvorenom ljuskom}

\section{P. Bischof}

Ispitana je elektronska struktura velikog broja organskih radikala pomoću neograničene verzije semiempirijske $\mathrm{MINDO} / 3$ metode. Dan je pregled dobivenih rezultata.

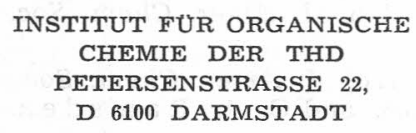

